\title{
European Society for Paediatric Nephrology
}

\author{
Twelfth Annual Meeting, Israel, 17-22 September 1978
}

\begin{abstract}
Amyloid nephropathy in familial Mediterranean fever. D. Zemer, M. Pras, and H. Boichis. Tel-Hashomer. Israel.

Familial Mediterranean fever, common among Sephardic Israelis, usually starts in childhood, with a $90 \%$ mortality by age 40 , mainly from amyloid nephropathy. Of 1000 patients given daily colchicine since 1972, none of the 968 who had no proteinuria initially has developed amyloid. Of 32 nephropathic patients treated for 2 years, the condition of 14 has improved, 6 remain static, and 12 have deteriorated.
\end{abstract}

Nephronophthisis (N): a review. A. Drukker. Jerusalem, Israel.

Nephronophthisis (N) and medullary cystic disease are probably one disease. Genetic differences remain unclear because criteria for the heterozygous state are lacking. $\mathbf{N}$ is not a primary cystic but a renal interstitial disease. Morphological and functional evidence favour a primary tubulopathy, perhaps proximal. A co-operative effort is needed to evaluate the origin of $\mathrm{N}$.

Ocular abnormalities in nephronophthisis (N). Th. Lennert, K. Scharer, and M. Brandis. Berlin, Heidelberg, and Hannover, FRG.

10 of 20 cases of nephronophthisis $(\mathrm{N})$ showed ocular abnormalities; 5 had tapeto-retinal degeneration, 3 nystagmus, 2 amblyopia, 1 cataract, 1 strabismus, 1 myopia, and 1 hypertensive retinopathy. Autosomal dominant inherited medullar cystic disease (MCD) is not associated with eye abnormalities. $\mathrm{N}$ is indistinguishable from Senior-Loken syndrome but may be different from MCD.

Epidemiology of kidney diseases in Finland in 1970-74. R. Lappi. Helsinki, Finland.

All patients treated in hospital in 1970-74 were analysed; 3 patients per 100000 population had urinary disease; $12 \%$ were $<15$ years of age, and $6 \%<5$ years. $90 \%$ of all patients had pyelonephritis or urological disease, but nephrosis, nephritis, and congenital malformations were more common in children.

Risk factors for cardiovascular diseases in children. F. Dorner, A. Spahr, R. Gautier, and J. P. Guignard. Lausanne and Sion, Switzerland.
1000 children aged 5,7 , and 10 years and 200 parents were examined. Obese children had higher blood pressures (BPs) than controls; no correlation between hypercholesterolaemia and BP was found. BPs were higher in parents of children with raised diastolic BPs. No severe hypertension was found; $5 \cdot 2 \%$ of children had proteinuria and $1.9 \%$ had haematuria. Routine screening is unjustified but obese children and those with a family history should be examined.

'Compensatory hypertrophy' of renal size in children with chronic pyelonephritis and unilateral renal damage. I. Claësson, B. Jacobsson, and J. Winberg. Gothenburg, Sweden.

26 randomly selected children with unilateral renal scarring from chronic pyelonephritis were followed up for 11 years. 16 had compensatory hypertrophy of the contralateral kidney but ipsilateral hypertrophy after damage was rare. Estimates of total renal area from radiographs suggested that the hypertrophy compensated for much of the renal damage and this compensatory growth accelerated in adolescence.

Syndrome of inappropriate ADH secretion in mechanically-ventilated newborn infants. J. Nutman, E. Wilunsky, and S. H. Reisner. Petah Tiqva, Israel. Three of 106 infants admitted to the neonatal intensive care nursery over 6 months had the syndrome of inappropriate ADH secretion diagnosed from hyponatraemia, weight gain, and highly concentrated urine. All were on mechanical ventilation, onset was rapid, and deep coma occurred in one infant. Fluid restriction and sodium replacement led to rapid improvement.

Cellular localisation and time course activity of alkaline and acid phosphatases in the nephrotic rat kidney. J. J. Helwig, C. Bollack, and J. Geisert, Strasbourg, France.

Nephrotic syndrome was induced by puromycin injection, proteinuria occurred on day 5 and was maximal on day 9. Alkaline phosphatase in tubules and medulla rose on day 1 and was at its maximum on day 5 , but was unchanged in glomeruli and cortex. Acid phosphatase was greater in glomeruli on day 1 but rose on days 6 and 7 in glomeruli and 
tubules. It is not clear whether these changes correlate with the renal disease or are a clinical effect of puromycin.

Renal cystic disease. J. Bernstein. Michigan, USA. Infantile polycystic disease (IPCD) and adult polycystic disease (APCD) may present at birth but can be distinguished clinically, radiologically, morphologically, and genetically. The hallmark of IPCD is medullary ductal ectasia, and in APCD there is irregular cyst distribution with severe glomerular involvement; cysts are commonly surrounded by smooth muscle fibres. Cysts may be secondary manifestations of heritable metabolic defects rather than inherent themselves. Tuberose sclerosis is an important differential diagnosis in a child with cystic disease.

Podocyte degeneration in glomerular disease. J. Churg and E. Grishman. New York, USA.

Degeneration of glomerular epithelial cell podocytes is common in many renal diseases. Either the original cell or a new cell can regenerate, adding in a haphazard fashion to the thickness of the basement membrane. Similar changes are seen in animals given puromycin or diacetylbenzidine, suggesting that podocyte damage is of toxic origin; impairment of capillary circulation may be a factor. The denudation of the basement membrane may account for the proteinuria.

Recent advances in congenital nephrosis of Finnish type (CNF). J. Rapola. Helsinki, Finland.

The incidence of congenital nephrosis of Finnish type (CNF) in Finland is 12 per million population per year and 6-8 new cases occur annually. Amniocentesis was performed on 23 'at risk' pregnancies, and in 7 patients, raised levels of amniotic alpha fetoprotein were found. Electron microscopical examination of kidneys from the aborted fetus confirmed the diagnosis in each case. Glomerular basement membranes in CNF show a decrease in collagen and 3-hydroxyproline of collagen, but the significance of these changes is not yet understood.

Prognosis of haemolytic-uraemia syndrome in children. M. F. Gagnadoux and R. Habib. Paris, France.

Between 1967 and 1976, 71 children with haemolyticuraemia syndrome were studied. The incidence of terminal renal failure was $12 \%$ in infants and $54 \%$ in older children. Renal biopsy within one month of onset and a year later in 22 children showed that prognosis was related to the number of glomeruli affected and whether the child had cortical necrosis; the development of severe hypertension correlated with the severity of vascular changes. The biopsy appearances were unique with progression to sclerosis of affected glomeruli.

Incidence, primary renal disease, and growth of children less than 5 years of age treated by regular dialysis and transplant. K. Scharer. Heidelberg, FRG. 87 children less than 5 years at start of treatment were registered with the EDTA (European Dialysis and Transplant Association) in 1977. Survival at one year was $90 \%$ and at 2 years it was $77 \%$, similar to older children. Nearly half the children lived in France and accounted for $10 \%$ of children treated there. Haemolytic-uraemia syndrome and cortical necrosis were important causes of renal failure. Growth was similar to that of older children.

Amino-acid metabolism and growth in infants with chronic renal failure. C. Giordano. Naples, Italy.

Daily weight gain was studied in infants with chronic renal failure on low-protein diets with supplements of different essential amino-acids (EAAs) and keto-acids. Marked improvement was demonstrated especially with EAAs but the formula of the EAA supplement used was critical.

Carbohydrate and lipid metabolism in chronic renal failure. C. Chantler. London, UK.

Energy metabolism is disturbed in renal failure with failure of normal glucose metabolism leading to potential cellular energy deficiency, protein malnutrition, and hyperlipidaemia. Low-protein diets with amino-acid supplements theoretically may ameliorate this but must be supplemented with energy; polyunsaturated fats should be used to reduce carbohydrate intake if hyperlipidaemia occurs.

Survey of the experience of the French paediatric units with dialysis and transplant programmes in children under 5 years of age. M. Broyer. Paris, France.

Since 1969,32 children ( 4 months $-4 \frac{1}{2}$ years) have been accepted for dialysis and transplant programmes in 6 paediatric units. Chronic haemodialysis (CHD) was generally performed with radial or humeral internal fistulae. Chronic peritoneal dialysis (CPD) was used alternately with CHD when vascular access was not available. 11 cadaver kidney transplants were done. 11 children have died, 9 are living with kidney transplants, 10 remain on CHD, and 2 on CPD at home. Survival was $74 \%, 70 \%$, and $62 \%$ at 1,2 , and 3 years.

Renal transplantation in children aged 1 to 5 years. S. M. Mauer. Minneapolis, USA.

Results of renal transplants in young children are sufficiently good compared with children of other ages for treatment to be offered to such children. 
Incidence of chronic renal faliure in Swedish children. I. Helin and J. Winberg. Malmo, Stockholm, Sweden.

To be published in full elsewhere.

Dialysis bone disease in childhood: treatment with 25 hydroxycholecalciferol (25OHD3). R. Dumas, $\mathrm{J}-\mathrm{Cl}$. Luciani, and P. Meunier. Montpellier, France. Five children on haemodialysis were treated with $25 \mathrm{OHD}_{3}$. Plasma calcium rose excessively in one child; growth velocity, plasma parathyroid hormone levels, and histological lesions of osteitis fibrosa cystica were unchanged. The osteomalacia present in one child improved and in 2 children a decrease in trabecular bone volume was observed.

Effects of various protein diets on growth, renal function, and survival of uraemic rats. I. Salusky, C. Kleinknecht, and M. Broyer. Paris, France.

Low-protein diet has been incriminated in growth failure in ureamia. In uraemic rats, high-protein diets $(37 \%)$ and $(27 \%)$ had deleterious effects on growth and survival, and led to progression to renal failure, compared with a diet limited to minimal requirements $(14 \%)$. It is important to avoid any protein excess in chronic uraemia.

Effect of anabolic steroids on growth in boys with chronic renal failure on haemodialysis. J. E. Carter, J. Burke, M. El-Bishti, R. Counahan, V. Wass, N. Dalton, and C. Chantler. London, UK.

Nine boys were treated with oxandrolone; in one the drug was stopped because of a rise in liver enzymes. Height velocity improved significantly and the children grew more rapidly than the advance in bone age. Intracellular water (cell mass) increased. Improvement in glucose tolerance and plasma branch chain amino-acids was observed.

Delayed puberty in males with chronic renal failure. P. Saenger, J. Ferraris, L. S. Levine, S. Pang, M. I. New, and J. E. Lewy. New York, USA.

31 boys aged 11 to 20 years were studied. Testosterone, dehydrotestosterone, and luteinising hormone levels were appropriate for pubertal status, suggesting normal Leydig cell function and activity of $5 \propto$ reductase enzyme. FSH levels were raised, suggesting damage to germinal eptihelium but they became normal after successful transplants.

Serum ferritin (SF) concentrations in children with chronic renal failure. D. E. Muller-Wiefel, E. Wolf, D. Schönberg, and K. Schärer. Heidelberg, FRG. Serum ferritin (SF) levels were determined in 32 children with chronic renal failure, 9 children on dialysis, 8 children with transplants, and 25 controls.
The level of SF was raised in all groups compared with controls. SF should be measured before starting iron treatment in children with renal failure because few of them are iron deficient.

Echocardiographic investigation of cardiac action during haemodialysis of children. J. R. Pfefferkorn, L. Diekmann, and U. Schulten-Baumer. Murster, FRG.

12 children on regular haemodialysis were examined. All showed increases in interventricular septum and posterior left ventricular wall due to myocardial hypertrophy. End diastolic diameter of left ventricle was increased, suggesting dilatation, but this decreased significantly during dialysis with fluid removal.

Dialysis and transplantation results in Europe 1977: review of the data from the paediatric registry of the EDTA. R. A. Donckerwolcke, C. Chantler, F. P. Brunner, H. J. Gurland, R. A. Hathway, C. Jacobs, H. Brygner, N. H. Selwood, and A. J. Wing. Holland, UK, Switzerland, FRG, France, and Sweden.

Results were published in Proceedings of the European Dialysis and Transplant Association, Volume 15, (1978) Pitmans Medical: London.

Mediation and effects of intrarenal fibrin deposition. P. F. Naish. Stoke, UK.

Intrarenal fibrin deposition may result from primary glomerular inflammation, or microvascular damage with or without intravascular coagulation. Defibrination is associated with protection from glomerular damage. Studies in nephrotoxic nephritis and endotoxin infusion in rabbits indicate the central role of neutrophils in fibrin deposition. Studies of neutrophils, platelets, and coagulation in patients may enable rational treatments to be developed.

Platelets and glomerulonephritis. J. S. Cameron. London, UK.

Platelets are important in the pathogenesis of glomerular damage from chronic soluble complex disease; antiplatelet agents may have a role in treatment but more studies using new tests of platelet function-such as serotonin levels-are needed to confirm this.

Combination therapy for mesangiocapillary nephritis in childhood. S. J. Chapman, J. S. Cameron, C. Chantler, and D. R. Turner. London, UK.

11 children with mesangiocapillary nephritis were treated with prednisolone, azothioprine, heparin, or warfarin; 7 children had a significant rise in glomerular filtration rate (GFR), in 2 the declining GFR stabilised, and 2 in terminal renal failure did 
not improve. In 2 children there was a rapid fall in GFR after withdrawal of immunosuppression.

Pathogenesis of focal glomerular hyalinosis and sclerosis. J. D. Elema, M. S. Schilthuis, and J. Grond. Groningen, The Netherlands.

From clinico-pathology observations in children with a variety of glomerular diseases and from experimental studies in rats, it is suggested that focal segmental hyalinosis and sclerosis is a nonspecific lesion. Local haemodynamic factors (pressure, flow) and changes related to the existence of nephrosis (hyperlipidemia, hypercoagulability) are pathogenetically involved although to different extents in different circumstances.

Immunological studies in the haemolytic-uraemia syndrome. L. Monnens, M. de Jong, P. van Muster, and J. Molenaar. Nymegen, Amsterdam, The Netherlands. To be published elsewhere.

Renal function and biopsy studies in patients with IgA and poststreptococcal glomerulonephritis. A one-year follow-up. T. Linne, A. Aperia, and $O$. Broberger. Stockholm, Sweden.

The renal functional changes of $\operatorname{IgA}$ and poststreptococcal glomerulonephritis were similar. GFR was depressed initially but became normal during the first months of the disease. The natriuretic response to an isotonic saline infusion was low in the early course of the disease, and took longer to become normal than GFR.

Deficiency of the second component of complement (C2D) with membranoproliferative glomerulonephritis. Recurrence after transplant. C. Loirat, C. Blum, M. Broyer, and M. Levy. Paris, France.

Membranoproliferative glomerulonephritis with subendothelial deposits was observed in a C2D boy without systemic disease. Immunofluorescence showed $\mathrm{IgC}, \mathrm{Clq}$, and $\mathrm{C} 4$ as granular deposits along capillary walls and within the mesangium. $\mathrm{C} 3$ was also deposited along capillary walls. The clinical course was severe. Recurrence of the glomerular lesions was observed 9 months after transplant.

Nonfamilial recurrent haematuria. L. Callis, M. Carreras, E. Vilaplana, and F. Castello. Barcelona, Spain.

Five girls aged 5-11 years had recurrent haematuria. Family history, renal function, complement levels, hearing, and renal biopsy evaluation by light microscopy and immunofluorescence were normal. Electron microscopical examination showed deposits within the basement membrane or thickening of the basement membrane with layering. The latter changes, present in 2 children, may represent a variant of Alport's syndrome.

Nephropathy in cyanotic congenital heart disease. J. P. Dommergues, M. Levy, M. C. Gubler, R. Habib, and V. Courtecuisse. Paris, France.

Three patients with congenital heart disease had a low glomerular filtration rate, haematuria, and proteinuria. Renal biopsy showed mesangial proliferation, and a double contour thickening of basement membrane due to a heterogenous deposit on the endothelial surface. Immunofluorescence was negative. Renal function improved after successful surgery and the glomerular changes disappeared in one case.

Role of circulating immune complexes (IC) in renal disease. R. J. Levinsky. London, UK.

Immune complexes (IC) differ in immunoglobulin class, subclass, size, complement binding, and antigen, and these differences account for the different results of the several tests used for detecting them. Size of IC is important in the pathogenicity of IC in lupus nephritis; the nonlocalisation of IC in steroid-sensitive nephrosis is probably due to the failure of the IC to bind complement. IgA IC are found in Henoch-Schoenlein disease but nephritis only occurs if IgG IC are present as well. The production of different IC may depend on the genetic differences of the host's immune response to antigen and may underlie the susceptibility to disease.

Complement profiles and $\mathrm{C3}$ nephritic factor-like activity (C3NeF-L) in children presenting with acute glomerulonephritis or membranoproliferative glomerulonephritis (MPGN). M. Levy, M. Sich, E. Pirotsky, and R. Habib. Paris, France.

Activation of the classical pathway was demonstrated in acute glomerulonephritis (34 patients) and membranoproliferative glomerulonephritis (MPGN) type 1 (20 patients) with C3NeF-L (Clinical Immunology and Immunopathology, 1978, 10, 477) in 9 and 7 respectively of these patients. Conversely, activation of the alternative pathway was documented in 13 patients with MPGN type 11, 10 of whom had C3NeF-L.

Complement activating factor (C3AF) in membranoproliferative glomerulonephritis. E. J. Lewis, B. G. Bartlow, and J. L. Roberts. Chicago, USA.

A factor capable of activating C3 (C3AF) by the alternative pathway was isolated by cryoprecipitation from a patient with type 1 membranoproliferative glomerulonephritis. Unlike $\mathrm{C} 3$ nephritic factor $\mathrm{C} 3 \mathrm{AF}$ is not an IgG; it is suggested that C3AF activates C3 by interaction with $\beta 1 \mathrm{H}$ leading to increased activity of the 'amplification feedback loop'. 
Kinetics of glomerular visceral epithelial cell phagocytosis of macromolecular aggregates from the lamina rara externa of the glomerular basement membrane. Z. Sharon and M. M. Schwartz. Illinois, USA.

The sequential intravenous administration of protamine and heparin results in aggregation and deposition of protamine-heparin aggregates (PHAgs) in the lamina rara externa of the glomerular basement membrane. Experiments in rats confirmed that removal of HPAgs is accomplished by epithelial cell phagocytosis by first-order kinetics unrelated to GFR. Epithelial cell removal of immune aggregates may be important in acute poststreptococcal nephritis and perhaps in other glomerular lesions with subepithelial deposition of immune complexes.

\begin{abstract}
Alternate-day v. intermittent prednisone in frequently relapsing nephrotic syndrome. J. Brodehl and $H$. P. Krohn. Hannover, FRG.

A prospective study was performed in 48 children with frequently relapsing nephrotic syndrome to compare alternate-day prednisone ( 23 children) $\left(35 \mathrm{mg} / \mathrm{m}^{2}\right.$ per $\left.48 \mathrm{~h}\right)$ with intermittent prednisone ( 25 children) $\left(40 \mathrm{mg} / \mathrm{m}^{2}\right.$ in $3 / 7$ days) during 6 -months' treatment. Results show that alternate-day prednisone is significantly more effective than equal amounts of intermittent prednisone for preventing patients from relapsing and for reducing the number of relapses.
\end{abstract}

Spontaneous remission and delayed therapy in steroid-sensitive nephrotic syndrome. R. J. Postlethwaite, I. B. Houston, and P. C. Powell. Manchester. UK.

To be published in full elsewhere.

Nephrotic syndrome. Value of new methods in kidney biopsy specimens. K. Kuehn, M. Brandis, R. Castelucci, and E. Reale. Hannover, Germany.

Ultrastructural investigation by scanning electronmicroscopy and freeze-fractured preparations of renal biopsies from children with minimal change nephropathy showed swelling of capillary epithelium, and widening and loss of peripheral foot processes, without real fusion.

Allergo-immunologic testing in children with nephrotic syndrome due to minimal change (MC) and focal glomerular sclerosis (FGS). D. E. Muller-Wiefel, U. Wahn, V. Lenhard, and W. Rebien. Heidelberg, FRG.

Atopic history score and serum IgE levels were similar in minimal change (MC), focal glomerular sclerosis (FGS), and controls, but 4 children with
MC had high levels. The proportion of positive prick tests and increased IgE antibodies was similar in the three groups but lymphocyte stimulation was depressed in MC, with intermediate results in FGS. One child with atopic MC, out of 3 desensitised to grass pollen, entered a stable remission, Allergic mechanisms play a role in only a few children with MC.

Nephrotic syndrome associated with tubular disorders in 5 children. Ph. Barthe and F. Bouissou. Toulouse, France.

Five children with steroid-resistant nephrotic syndrome developed mixed tubular acidosis (5/5), hypokalaemia $(4 / 5)$, glycosuria $(5 / 5)$, hypophosphataemia $(2 / 5)$, aminoaciduria $(2 / 5)$, and tubular proteinuria $(2 / 5)$. Progression to renal failure occurred and repeat biopsy showed focal glomerular sclerosis.

Distal renal tubular acidosis (DRTA) in 23 infants and children. M. Dechaux, M. Broyer, G. Lenoir, and P. Guesry. Paris, France.

23 infants and children with primary distal renal tubular acidosis (DTRA) were studied; one had bicarbonate wasting, 3 had no systemic acidosis. Deafness was noted in 5. Dehydration and vomiting were early signs, nephrocalcinosis was common with hypercalaemia. Concentrating capacity was reduced in 15 children. Growth improved with treatment. Familial factors were present in 7 children. DTRA is not a homogenous disorder.

Adolescent cystinosis. Case report and review of the literature. G. Knopfle, H-P Weber, and E. Harms. Bonn, Heidelberg, FRG.

Cystinosis was diagnosed in a 13-year-old boy with a Fanconi's syndrome and a glomerular filtration rate of $34 \mathrm{ml} / \mathrm{min}$ per $1 \cdot 73 \mathrm{~m}^{2}$. Growth and development were normal. Progression to renal failure occurred over 5 years. Adolescent cystinosis is associated with glomerular proteinuria, lower intracellular cystine than in the infantile form, and slower progression.

Physical chemical basis of urinary stone disease. W. G. Robertson, M. Peacock, R. W. Marshall, and B. E. C. Nordin. Leeds, UK.

Abnormal crystalluria leading to stone disease results from imbalance between saturation of urine and inhibitory activity which prevents large aggregates; $\mathrm{pH}$, calcium oxalate, acid mucopolysaccharide, and uric acid are important factors in this balance. The relative contribution of these risk factors enables the probability of stone formation in an individual to be calculated. 
Idiopathic hypercalciuria (IH)—an old enigma. U.A. Liberman. Petah Tiqva, Israel.

There appear to be 4 varieties of idiopathic hypercalciuria (IH): (1) increased intestinal calcium absorption, (2) decreased tubular calcium reabsorption, (3) normocalcaemic hyperparathyroidism, and (4) decreased tubular phosphate reabsorption. The aetiology of these forms of IH remains obscure.

Idiopathic hypercalciuria in childhood: clinical and metabolic studies. M. Tieder, H. Stark, and R. Shainkin-Kerstenbaum. Beer-Sheba, Israel.

Compared with 10 controls, 3 children with IH demonstrated evidence of: (1) increased intestinal calcium absorption, (2) decreased parathyroid (PTH) and increased calcitonin (CT) activity, (3) low plasma phosphate due to low TmP/GFR (phosphate reabsorption). These suggest that low TmP/GFR is primary, causing low $\mathrm{Pp}$, which stimulates 1.25 $(\mathrm{OH})_{2}$ vitamin $\mathrm{D}$ production and increased $\mathrm{Ca}$ absorption. This, with resulting low PTH and high CT, causes hypercalciuria.

Hypercalciuria (HC), nephrocalcinosis, and nephrolithiasis in metabolic disorders. $O$. Mehls, $F$. Manz, and H. Schmidt. Heidelberg, FRG.

Hypercalciuria (HC) secondary to hypercalcaemia is seen with vitamin D intoxication. HC due to failure of proximal tubular reabsorption occurs in the Fanconi's syndrome in association with decreased sodium reabsorption; magnesium supplements to correct hypomagnesaemia may cause $\mathrm{HC}$ by competing for reabsorption. In renal tubular acidosis $\mathrm{HC}$ is secondary to the metabolic acidosis. Hyperoxaluria may cause nephrolithiasis and 5 of 70 children with phenylketonuria (PKU) developed stones; hyperoxaluria occurs in some children with PKU and occasionally with tyrosinosis.

Address of authors may be obtained from Dr C. Chantler, Evelina Department of Paediatrics, Guy's Hospital, St Thomas Street, London SE1 9RT. 